\title{
Asymmetric angular emission in semiconductor microcavities
}

\author{
P. G. Savvidis, ${ }^{1}$ J. J. Baumberg, ${ }^{1}$ R. M. Stevenson, ${ }^{2}$ M. S. Skolnick, ${ }^{2}$ D. M. Whittaker,${ }^{3}$ and J. S. Roberts ${ }^{4}$ \\ ${ }^{1}$ Department of Physics \& Astronomy, University of Southampton, SO17 1BJ, United Kingdom \\ ${ }^{2}$ Department of Physics, University of Sheffield, Sheffield S3 7RH, United Kingdom \\ ${ }^{3}$ Toshiba Research Europe Ltd, Cambridge, CB4 4WE, United Kingdom \\ ${ }^{4}$ Department of Electronic and Electrical Engineering, University of Sheffield, Sheffield, S1 3JD, United Kingdom
}

(Received 22 February 2000; revised manuscript received 5 June 2000)

\begin{abstract}
Strongly angular-dependent emission properties are observed from a semiconductor microcavity pumped along a critical angle of incidence. In contrast to the luminescence from conventional semiconductor heterostructures, the emission is completely asymmetrical with respect to the sample normal. The results imply that parametric scattering dominates the energy relaxation of polaritons, and is enhanced by the deformed shape of the dispersion relations.
\end{abstract}

Planar semiconductor microcavities are of considerable interest because they can be used to modify and enhance optical emission from embedded semiconductor heterostructures. Highly controlled light output from microcavity lightemitting diodes (LEDs) and vertical cavity surface emitting lasers (VCSELs) is of practical application, ${ }^{1}$ while organic microcavities have also successfully supported laser operation. $^{2}$ When cooled sufficiently, optimally designed semiconductor microcavities enter a regime of strong coupling in which the exciton resonance mixes sufficiently with the cavity photon mode to form two new polariton modes. ${ }^{3}$ Recent publications highlight the nonlinear power dependence of the luminescence from such samples and have controversially attributed it to stimulated scattering. ${ }^{4-7}$ However under nonresonant pumping, light from these heterostructures, as from bare quantum wells, remains symmetrically distributed about the normal to the sample.

In this paper, we comprehensively characterize both spectrally and angularly, the emission of light from microcavity samples which are resonantly pumped at a critical angle. In this regime strong angular asymmetries are seen in the photoluminescence, produced by parametric scattering between pairs of polaritons with different in-plane momentum and energy. Strong nonlinearities in the emission are produced when this scattering is stimulated by occupation of the final polariton state. By simultaneously injecting a pulse of light incident normally, the parametric scattering is externally stimulated and the two final states clearly observed.

Angular measurements on these microcavity polaritons provide crucial information because they probe the whole range of exciton-photon interactions, starting from $0^{\circ}$ where the coupling is strong and reaching $\pm 55^{\circ}$ where the two modes are effectively not coupled. ${ }^{8}$ Our experimental geometry concentrates on resonant pulsed excitation at the point of inflection of the lower polariton dispersion $\left(k_{\|}=2.1 \times 10^{6}\right.$ $\mathrm{m}^{-1}$ ), while detecting the time-integrated luminescence emerging at different angles (Fig. 1). When the exciton and photon modes are degenerate at $\theta=0^{\circ}\left(\omega_{\text {ex }}=\omega_{\text {cav }}\right)$, this pump configuration generates parametric scattering shown by arrows in Fig. 2(a), in which pump polariton pairs at $\left(\omega_{p}, \mathbf{k}_{p}\right)$ end up at both $\left(\omega_{p} \pm \epsilon, \mathbf{k} \pm \mathbf{q}\right){ }^{9}$ To demonstrate the strong sensitivity of this scattering rate to the final state population, a normally incident probe pulse can be set to arrive simultaneously with the pump pulse. In order to inject polaritons at specific $(\omega, \mathbf{k})$, the optical pulses are spectrally filtered and aligned along particular incident angles, without grossly modifying their time of arrival at the sample. To achieve this we constructed the femtosecond-stable goniometer shown in Fig. 1, which rotates under the center of the sample. The arms for pump, probe, and detection beams each allow access to $\theta \leqslant \pm 80^{\circ}$. A $100-\mathrm{fs}$ Ti:S laser is used to generate the different 3 -ps pulses which are shaped in the spectral plane using computer-controlled liquid crystal modulators. ${ }^{10}$ The pump powers are kept sufficiently low so that the exciton oscillator strength suffers no appreciable bleaching. ${ }^{11}$ The emitted light from the sample is collected in a detection cone of $\pm 0.14^{\circ}$ and is coupled through polarization-sensitive optics into a multimode fiber. To avoid averaging across regions of different excitation intensity, only the inner part $(50 \mu \mathrm{m})$ of the PL spot is sampled. A monochromator with nitrogen-cooled charge-coupled device (CCD) of spectral resolution $0.035 \mathrm{meV}$ is used to analyze the time-integrated emitted light.

The sample studied here is a $3 \lambda / 2$ semiconductor microcavity grown by metal organic vapor phase epitaxy (MOVPE) and consists of two pairs of three 100- $\AA$

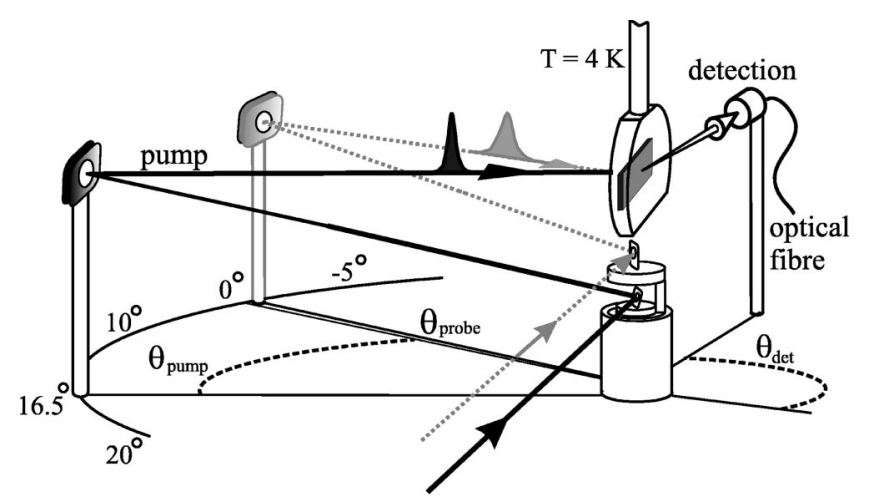

FIG. 1. (a) Femtosecond-stable goniometer for time- and angleresolved pump-probe experiments. Relative time delay is unchanged (within $\sim 50 \mathrm{fs}$ ) as pump or probe angles are scanned up to $\pm 80^{\circ}$. 

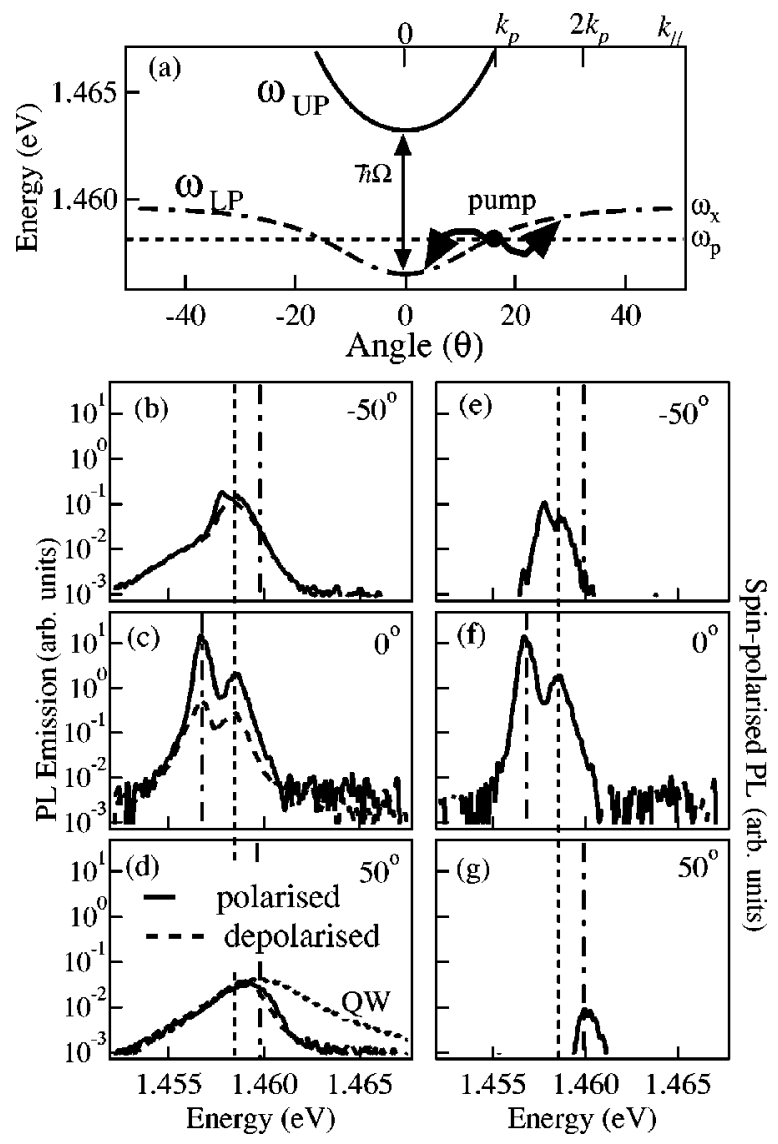

FIG. 2. (a) Lower (dot-dashed) and upper (solid) polariton dispersion relation. The resonantly tuned pump pulse injected at $16.5^{\circ}$ produces parametric scattering shown by arrows. Dotted line marks elastic pump scatter. (b) -(d): PL spectra at $-50^{\circ}, 0^{\circ}, 50^{\circ}$ for both $(+: \pm)$ polarization configurations. The pump power is 22 $\mathrm{W} \mathrm{cm}^{-2}$. Bare QW PL also shown in (d). (e)-(g): Spin-preserving PL emission extracted from (b) $-(d)$.

$\mathrm{In}_{0.06} \mathrm{Ga}_{0.94} \mathrm{As}$ QWs in 100- $\AA$ GaAs barriers, surrounded by distributed Bragg reflectors (DBRs). ${ }^{9}$ The sample strip is oriented with the cavity thickness constant in the plane formed by the incident beams. Figure 2(a) shows the dispersion relation of the two polariton modes with normal mode splitting of $\hbar \Omega \sim 7 \mathrm{meV}\left(\right.$ at $\theta=0^{\circ}$ ). The sample is held in a cold finger, wide field-of-view cryostat at a temperature of $4 \mathrm{~K}$. Polaritons are resonantly injected by a pulse of light tuned to match the lower polariton dispersion at $16.5^{\circ}$ from a spectrally filtered pump pulse $\left(\leqslant 30 \mathrm{~W} / \mathrm{cm}^{2}\right)$ of $2 \mathrm{meV}$ bandwidth focused on a $100 \mu \mathrm{m}$ spot.

We first investigate the scattering mechanisms on the lower polariton branch by measuring angular-, spin-, and wavelength-resolved photoluminescence (PL) over a large range of angles $\left( \pm 55^{\circ}\right)$. Spin-selectivity distinguishes the fast scattering mechanisms preserving spin polarization, from slow depolarizing relaxation processes. We use a circularly polarized $\sigma^{+}$pump beam for excitation ${ }^{12}$ and detect both circular polarizations $\sigma^{+}, \sigma^{-}$of the emitted light. We use the spin notation (Pump:Detection) $=(+: \pm)$. Figures 2(b) $-2(d)$ show the emission spectra for polarized PL $(+:+)$ and depolarized $\operatorname{PL}(+:-)$ configurations at $\theta_{d e t}=-50^{\circ}$, $0^{\circ}, 50^{\circ}$ on the same logarithmic scale. The difference $\Delta \mathrm{PL}=\mathrm{PL}(+:+)-\mathrm{PL}(+:-)$, between the solid and dashed curves [Figs. 2(e)-2(g)] corresponds to the spin-preserving part of the scattering, and is dominated by the polarized component. Previously it has been shown that for nonresonant excitation the PL spectrum at $T=77 \mathrm{~K}$ is thermalized and that absorption spectra are related to the PL spectra through a Boltzmann factor. ${ }^{13}$ This picture is very different at the low $T$ here $(k T \ll \hbar \Omega)$ when excitation is resonant. Thermalization is not achieved and selective energy and momentum scattering is observed. However no emission is seen at the upper polariton throughout this regime, demonstrating the lack of significant carrier heating. The low energy tails of these excitonlike polaritons match those of a bare 100- $\AA$ $\mathrm{In}_{0.06} \mathrm{Ga}_{0.94}$ As QW sample [Fig. 2(d), dotted] and are attributed to the Urbach tail arising from localized exciton states. ${ }^{14}$ The fast spin-preserving polariton scattering predominates in the $k_{\|}=0$ region, but only for pump intensities $>5 \mathrm{~W} \mathrm{~cm}^{-2}$. The vertical dashed line is at the energy of elastic pump scattering, while the dash-dotted line marks the angledependent lower polariton energy. The data show that there is a pronounced asymmetry of the emission, even at relatively large angles $\pm 50^{\circ}$ where the exciton and photon are uncoupled.

For a full picture of the asymmetrical scattering we acquire PL spectra over the whole range of angles and both $(+: \pm)$ detection polarizations. Similar spectra are observed for linear field polarizations. Contour maps of the spinpreserving emission pattern, $\Delta \mathrm{PL}(\omega, \theta)$, at different angles provide information on the extent of the emission anisotropy [Fig. 3(a)]. The solid white curve shows the extracted peak position of the emission at each angle and is blue-shifted by $\sim 0.7 \mathrm{meV}$ as compared to the dispersion relation at low excitation powers (dashed line). The origin of this small blueshift will be deferred to a more extended publication, however it is clear that the polariton dispersion has not collapsed and the anticrossing persists. ${ }^{15}$ Direct transmission of the pump beam tuned at $1.458 \mathrm{eV}$ is seen at $\theta=+16.5^{\circ}$ $(\times)$. Figure 3(a) shows the significant difference in the emission spectra for positive and negative angles. Such an asymmetrical emission distribution has not to our knowledge been seen previously from a planar semiconductor heterostructure. The asymmetry is due to parametric scattering of specific polariton pairs, which then rapidly escape from the microcavity. The off-axis pump pulse breaks the cylindrical symmetry and the sample remembers this excitation condition, producing a characteristic signature in the photoluminescence.

The highly spin-polarized emission from the lower polariton branch seen for $\theta_{d e t}>0$, indicates the specific fast intrabranch scatterings that occur after resonant excitation. For positive angles the dispersion relation provides ideal energymomentum conditions for intrabranch polariton pair scattering [Fig. 2(a)]. This process preserves the polariton spin which is excited by the circularly polarized pump. On the other hand at negative angles $\theta_{d e t}<-5^{\circ}$ only a small amount of spin-polarized polariton emission emerges because intrabranch parametric scattering is suppressed by the imposed dispersion relation. All pairs of polariton-polariton scatterings for which energy and momentum is conserved can occur from the reservoir of polaritons injected by the pump. This restricts polariton scattering into only $\mathbf{k}$ states which lie along a radial line defined by the incident $\mathbf{k}_{p}$, and 


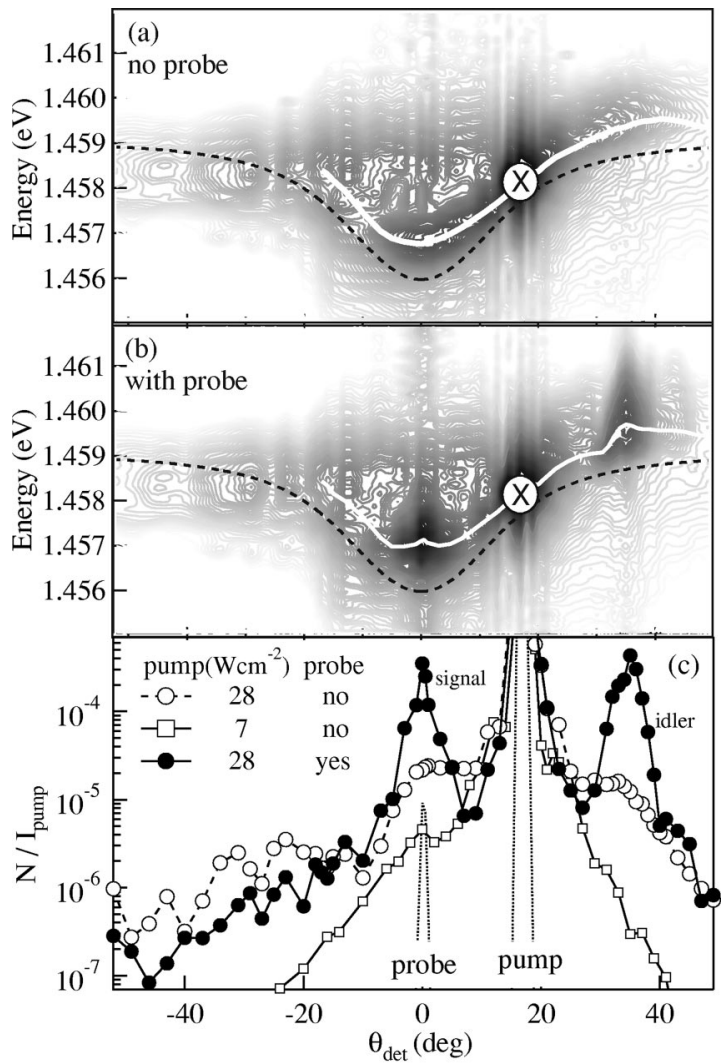

FIG. 3. Contour maps of the $\Delta \mathrm{PL}$ spectra vs angle for a 28 $\mathrm{W} \mathrm{cm}{ }^{-2}$ pump pulse at $16.5^{\circ}(\times)$. The white line marks the energy position of the peak PL, while the dotted line is the lower polariton dispersion. (a) Spontaneous and self-stimulated PL emission. (b) Probe-stimulated PL emission showing the signal gain at $0^{\circ}$ and idler beam at $35^{\circ}$. (c) Normalized lower branch polariton emission vs angle with ( ) and without probe $(\square, \bigcirc)$ for pump powers 7 , $28 \mathrm{~W} \mathrm{~cm}^{-2}$. Angular widths of pump and probe beams also plotted.

not into different directions in the $k$ plane. ${ }^{16}$ However the high $k_{\|}$polaritons close to $\omega_{x}$ experience much faster subsequent scattering into excitons at even higher $k_{\|}$where they cannot radiate. The radiative $\left(\Gamma_{c}\right)$ and exciton $\left(\Gamma_{x}\right)$ scattering rates then determine how much light escapes at each angle $\theta$, so the PL intensity detected depends on the population,

$$
N(\theta) \propto \operatorname{PL}(\theta)\left\{1+\frac{|X|^{2} \Gamma_{x}}{|C|^{2} \Gamma_{c}}\right\}=\operatorname{PL}(\theta)\left(1-\alpha+\alpha /|C|^{2}\right),
$$

for exciton and photon fractions $X, C$. Using the ratio $\alpha$ $=\Gamma_{x} / \Gamma_{c} \sim 0.55$, we convert the integrated PL along the lower polariton branch (measured over eight decades of intensity) into polariton populations $N(\theta) / I_{\text {pump }}$ normalized by the pump intensity in Fig. 3(c). This value of $\alpha$ is derived from the ratio of the total exciton linewidth to the cavity linewidth, consistent with a homogenous exciton broadening greater than the inhomogeneous linewidth contribution, and implying fast exciton scattering. Further work to investigate the influence of localized excitons and dark polaritons is in progress.

At low powers $\left(7 \mathrm{~W} \mathrm{~cm}^{-2}\right)$ the spontaneous scattering rate is low $(\square)$ but at higher powers $(\bigcirc)$ the polaritons relaxing down the dispersion curve act as a final state popu-

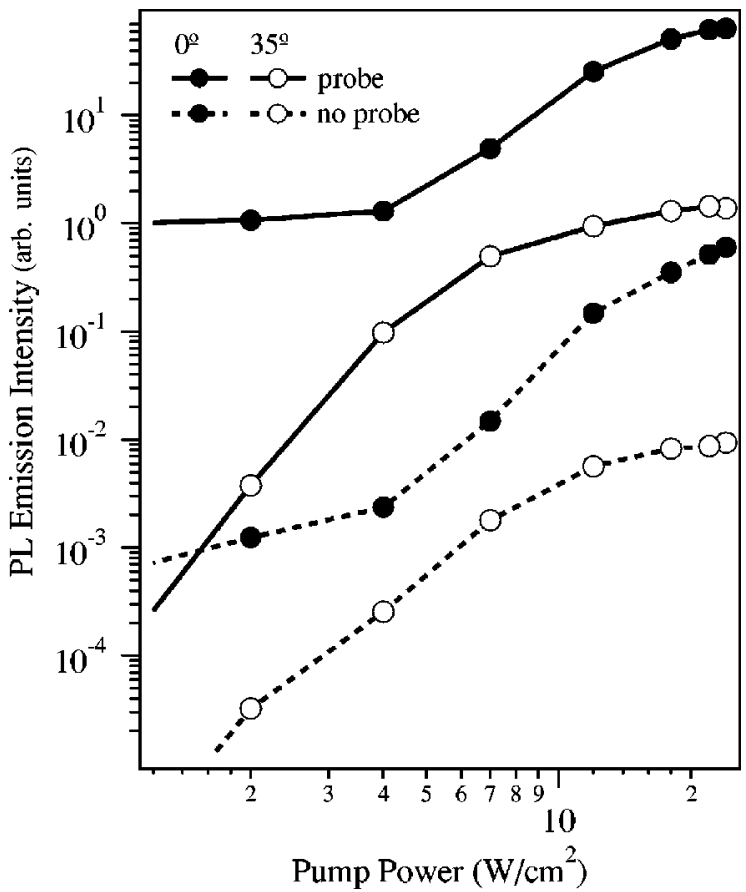

FIG. 4. Integrated lower polariton PL emission vs pump power at $0^{\circ}, 35^{\circ}$ both with and without the probe pulse. The emission is normalized to the transmitted probe power, with incident probe power of $400 \mathrm{~m} \mathrm{~W} \mathrm{~cm}^{-2}$.

lation to initiate further stimulated scattering into these states. Such stimulated parametric scattering has previously been seen as gain in pump-probe experiments, ${ }^{9}$ and depends on the bosonic exchange symmetry of the polaritons. Pair scattering gives equal populations symmetrically either side of the pump energy. Similar processes have been previously termed hyper-Raman scattering in studying bulk polaritons using $\mathrm{MW} \mathrm{cm}{ }^{-2}$ pulses. ${ }^{17}$ Additional emission is clearly resolved in the vicinity of the pump beam. Pair scattering is also responsible for this symmetric broadening and for the two correlated broad shoulders observed at $0^{\circ}$ and $35^{\circ}$ which correspond to the optimum phase matching condition. ${ }^{15}$

To confirm this parametric scattering process, a weak cocircularly polarized probe pulse is simultaneously injected into the $k_{\|}=0$ polaritons, dramatically modifying the emission pattern [Fig. 3(b)]. In contrast to the stimulated scattering induced by the pump alone, the probe beam now injects a final state population at a specific angle $\left(\theta=0^{\circ}\right)$ thus increasing the scattering rate according to

$$
\frac{d N_{\text {final }}}{d t} \propto\left(1+N_{\text {final }}\right) \times N_{\text {pump }}
$$

and provoking gain in the probe direction. The excess energy and momentum is taken away by a second pump polariton which is scattered to a higher "idler" angle and here clearly seen for the first time at $\theta=35^{\circ}$. The modified lower branch PL [@, Fig. 3(c)] shows how the injected probe pulse redistributes the light emitted at each angle. A significant reduction in the emission (up to $70 \%$ ) between $5-15^{\circ}$ and $20-30^{\circ}$ (confirmed by direct time-resolved transmission measurements) is caused by efficient probe-induced scattering into 
the $k_{\|}=0$ final state, which extracts most of the available pump polaritons before other allowed pair scattering processes can act.

It is instructive to compare the angular size of the gain and idler beams with incoming pump and probe angular beamwidths. Both gain and idler beams have the same angular width indicating that the momentum broadening observed is an inherent property of parametric scattering and is not caused by their very different local energy/momentum relaxation. The beams consist of two superimposed narrow and broad angular peaks. The narrow peak has the same $\sim 1^{\circ}$ angular width as the incident laser beams while the broad peak has $\Delta \theta \sim 4^{\circ}$. This cone of emission is controlled by the modified dispersion relation through the range of allowed parametric scatterings, and by the diffraction properties of polaritons in the Fabry-Pérot microcavity modes. ${ }^{18}$

Further confirmation of the origin of the emission asymmetry due to parametric scattering is provided by the correlated power dependence of gain and idler (Fig. 4). With no injected $k_{\|}=0$ polaritons, the PL emerging at normal incidence becomes superlinear in pump power at a threshold of 5 $\mathrm{W} \mathrm{cm}^{-2}$. Above the same threshold, the idler emission dramatically increases, and the two remain correlated, although the idler emission can be seen to saturate above $20 \mathrm{~W} \mathrm{~cm}^{-2}$. When the probe pulse injects an initial polariton population at $k_{\|}=0$, strong emission (gain) is only seen above the same pump threshold, while the idler emission at $35^{\circ}$ turns on at the same threshold power, before saturating. The reason for the consistent saturation in the idler emission is not clear, however it may be accounted for by a density-dependent scattering rate of idler polaritons into high- $k$ excitons, which in any case demands more detailed theoretical and experimental investigation.

In conclusion, the asymmetric angular nature of spectral emission from microcavities is characterized for the first time. We unambiguously show that PL of the lower polariton branch after resonant excitation at particular angles is dominated by stimulated parametric scattering, which is both manifestly nonequilibrium and asymmetric with respect to the sample normal. Injecting a probe pulse using a femtosecond goniometer reorganises the emission, generating a strongly-amplified signal in the probe direction, and a phasematched idler pulse at high angles, well described by parametric scattering.

J.J.B. acknowledges support from the University of Southampton, Coherent U.K. Ltd., Hitachi Europe Ltd., and fruitful discussions with B. Deveaud and C. Ciuti. This work was partly supported by EPSRC GR/M43890, GR/L32187, and HEFCE JR98SOBA, and EC CLERMONT, contract No. HPRN-CT-1999-00132.
${ }^{1}$ J. L. Jewell et al., IEEE J. Quantum Electron. 27, 1332 (1991).

${ }^{2}$ T. Granlund et al., Chem. Phys. Lett. 310, 577 (1999).

${ }^{3}$ C. Weisbuch, M. Nishioka, A. Ishikawa, and Y. Arakawa, Phys. Rev. Lett. 69, 3314 (1992).

${ }^{4}$ A. Imamoglu et al., Phys. Rev. A 53, 4250 (1996).

${ }^{5}$ P. Senellart and J. Bloch, Phys. Rev. Lett. 82, 1233 (1999).

${ }^{6}$ Le Si Dang et al., Phys. Rev. Lett. 81, 3920 (1998).

${ }^{7}$ F. Tassone and Y. Yamamoto, Phys. Rev. B 59, 10830 (1999);

A. I. Bobrysheva et al., Phys. Status Solidi B 212, 105 (1999).

${ }^{8}$ R. Houdré et al., Phys. Rev. Lett. 73, 2043 (1994).

${ }^{9}$ P. Savvidis et al., Phys. Rev. Lett. 84, 1547 (2000).

${ }^{10}$ A. Weiner et al., Opt. Lett. 15, 326 (1990).

${ }^{11}$ M. Kira et al., Phys. Rev. Lett. 79, 5170 (1997); X. Fan, H. Wang, H. Q. Hou, and B. E. Hammons, Phys. Rev. B 56, 15256 (1997).

${ }^{12}$ In order to compensate for Fresnel reflections, the pump beam must be elliptically polarized outside the sample to produce circular polarization in the plane of the quantum wells.

${ }^{13}$ R. P. Stanley, S. Pau, U. Oesterle, R. Houdré, and M. Ilegems, Phys. Rev. B 55, R4867 (1997).

${ }^{14}$ J. D. Dow and D. Redfield, Phys. Rev. B 5, 594 (1972).

${ }^{15}$ C. Ciuti et al., Phys. Rev. B 62, R4825 (2000); C. Ciuti et al., cond-mat/0008408 (unpublished).

${ }^{16}$ This is confirmed by images which show the emerging light lies along a line and not a cone: P. G. Savvidis et al., Phys. Status Solidi B 221, 77 (2000).

${ }^{17}$ B. Honerlage, R. Levy, J. B. Grun, C. Klingshirn, and K. Bohnert, Phys. Rep. 124, 161 (1985); S. Savasta and R. Girlanda, Phys. Rev. B 59, 15409 (1999).

${ }^{18}$ F. De Martini, F. Cairo, P. Mataloni, and F. Verzegnassi, Phys. Rev. A 46, 4220 (1992). 